\title{
Dendritic cell-glioma fusion activates T lymphocytes by elevating cytotoxic efficiency as an antitumor vaccine
}

\author{
FUMING TIAN ${ }^{1,2}$, CHANGWU DOU ${ }^{2}$, SONGTAO QI ${ }^{1}$, BO CHEN ${ }^{2}$, LIQUN ZHAO ${ }^{2}$, XIAOJUAN WANG ${ }^{3}$ \\ ${ }^{1}$ Department of Neurosurgery, Nanfang Hospital, Southern Medial University, Guangzhou, PR China \\ ${ }^{2}$ Department of Neurosurgery, First Affiliated Hospital of Inner Mongolia Medical University, Hohhot, Inner Mongolia \\ ${ }^{3}$ Guangdong Foshan Medical University, Foshan, Guangdong, PR China
}

\begin{abstract}
Background: Hybrid cells produced by fusions of tumor and dendritic cells (DC) have demonstrated remarkable efficacy for priming the anti-tumor immune response. In the current study, we examined the antitumor activity of cytotoxic T lymphocytes (CTLs) primed in response to a tumor vaccine comprising a glioma-DC fusion as part of a therapeutic against glioma.

Material and methods: Primary cultured glioma cells were fused with peripheral blood DC under conditions of polyethylene glycol (PEG) incubation. Glioma cell suspensions were designated as three groups to include (1) CTL-effective cell group activated by fused cells; (2) CTL-effective cell group stimulated by co-cultured glioma cells and DC cells; and (3) lymphocyte-only group as a control, which was not stimulated by the DC. Cytotoxicity of CTLs on glioma cells was accessed by MTT assay in vitro.

Results: Glioma cells with peripheral blood DC were cultured and fused. The killing effect of CTLs pre-activated by fused cells was significantly higher than that of the co-culture CTL group with unsensitized lymphocytes $(p<0.01)$. The killing activity, as measured by an enhanced efficiency ratio, was increased significantly in the co-cultures of fused cells with CTL groups $(p<0.01)$.

Conclusions: The glioma-dendritic cell fusion vaccine possessed a more effective anticancer activity by stimulating the effector activity of CTLs.
\end{abstract}

Key words: glioma cell, dendritic cell, cytotoxic T lymphocyte, dendritic cell/glioma fusion.

(Centr Eur J Immunol 2014; 39 (3): 265-270)

\section{Introduction}

Glioma, the most common malignant tumor of the central nervous system, presents an aggressive and therapeutic-resistant assault in cancer development [1]. Traditional treatments including surgery, radiotherapy and chemotherapy have shown an extremely low cure rate, with a high recurrence as well as poor prognosis. Prevention and treatment for recurrent glioma following radiotherapy and surgical resection are two fundamental strategies that need to be urgently optimized in the clinical setting [1]. In this regard, immunotherapy is considered a promising modality of the available cancer treatment regimens. Dendritic cells (DC) possess the most powerful antigen-presenting ability and play an important role in immunotherapy in glioma [2]. Dendritoma, the fusion tumor vaccine, acts not only as a tumor cell antigen, but also maintains the DC function of antigen presentation and activation of tumor-specific effector $\mathrm{T}$ lymphocytes, raising a new research field due to the promising findings [3].
Effective immunotherapy strategies against glioma have been demonstrated by developing antiglioma effector cytotoxic $\mathrm{T}$ cells primed in response to combined presentation to both interferon- $\beta$ gene vector and DC [4]. Dhodapkar et al. further indicated that targeting natural killer T (NKT) cells may provide a novel strategy for immunotherapy of glioma [5]. Animal studies comparing the co-administration of intratumoral injection of dendritic (DC) in combination with irradiated glioma cells (IR-GC) in mouse brain, demonstrated prolonged survival of brain tumor-bearing mice [6]. These results all support the therapeutic efficacy of intratumoral injection of DC and IR-GC. Overall, DC therapy has proven to be safe in both animal models and clinical trials for improved immunological microenvironment in the central nervous system (CNS) [7, 8].

Dendritic cell-based immunotherapy has historically utilized DC pulsed with tumor lysate or tumor-peptide antigens to induce an antitumor immune response. Results from related studies examining the T-cell stimulatory capacity against 
autologous human DC-glioma fusion or DC-loaded apoptotic glioma cells indicated an enhanced cytotoxicity and proliferation response against autologous tumor cells from non-adherent peripheral blood mononuclear cells [9]. In these results, the tumor-cytolytic response was induced by CD40 ligand. Hybrid cells produced by fusions of tumor and autologous DC have also demonstrated remarkable efficacy for stimulating an anti-tumor immune response in both preclinical and clinical studies of extra-cranial neoplasms [10]. Koido et al. summarized that the administration of DC/malignant cell fusions to cancer patients offers a safe, immunotherapeutic intervention strategy, with efficient tumor-specific T-cell responses in vitro [10]. However, the objective clinical responses to DC/cancer cell fusions, and the stability of DC/ malignant cell fusions in generating lasting effector cytotoxic $\mathrm{T}$ lymphocytes were rarely reported. Consequently, the mechanism of human DC-glioma fusion, which showed cytotoxicity against autologous tumor cells remains unclear. In the current study, we hypothesize that dendritic cell-glioma fusion may enhance the antitumor activity of cytotoxic T lymphocytes, providing improved results to confirm the immunogenicity of DC/glioma cell fusions as anticancer vaccines.

\section{Material and methods}

\section{Reagents}

Main reagents including rhGM-CSFrh-IL-4, rh-TNF- $\alpha$ (Strathmann Biotech, Hamburg, Germany), FITC-labeled mouse anti-human CD86, PE labeled HLA-DR mAb (Immunotech, Marseille, France), RPMI1640 (Gibco, Grand Island, NY, USA), FCS (Hangzhou Sijiqing Biological Engineering Materials, Hangzhou, Zhejiang, China), lymphocyte separation medium (Shanghai No. 2 Chemical Reagent Factory, Shanghai, China), MTT (Sigma, St. Louis, MO, USA), DMSO (Aibio Biotech, Shanghai, China), and PKH26 (Sigma, St. Louis, MO, USA).

\section{Specimens}

Fourteen cases of glioma patients, diagnosed pathologically (aged 14 to 57, mean 42; 4 males and 10 females), were selected from the Department of Neurosurgery, Inner Mongolia Medical University Hospital from October 2006 to September 2011. Of the fourteen cases, there were four cases of glioblastoma multiforme, seven cases of astrocytoma, and three cases of oligodendroglioma. Recruitment of patients and blood draw were approved by the Ethical Committee of the First Affiliated Hospital of Inner Mongolia Medical University.

\section{Dendritic cells separation, induction and phenotype detection}

Diluted anticoagulated peripheral blood $(100 \mathrm{ml})$ was mixed with an equal volume of PBS and centrifuged using lymphocyte separation medium (Ficoll-paque). Four layers were separated as red blood cells, lymphocyte separation medium, mononuclear cells (PBMC) and serum. The mononuclear cell layer was carefully drawn off, centrifuged, and resuspended in a complete culture medium following washing and culture. Non-adherent lymphocytic cells were collected after 2 hours of incubation, and then were frozen and kept in liquid nitrogen for subsequent use involved in the harvesting of purified T lymphocytes. To the adherent cells there was added a complete culture medium containing $1000 \mathrm{ng} / \mathrm{ml}$ each of rhGM-CSF and rhIL-4; half of the complete medium with the above cytokines was replaced after three days. After 6 days in culture, TNF- $\alpha$ (tumor necrosis factor $\alpha$ ) $50 \mathrm{ng} /$ $\mathrm{ml}$ was included in the culture media, and the DC were then collected at day 10 post harvest. CD86, HLA-DR phenotypic testing was performed at day 6 and 10, respectively.

\section{Glioma cells in primary culture and subculture}

Fresh glioma was aseptically removed from the patient under surgical conditions. Tissue was washed with RPMI1640, cut into sections for trypsin digestion, and filtered with steel mesh for preparation of glioma cell suspension. Cell suspension was then centrifuged and washed twice, and resuspended in a complete culture medium for incubation. Cells were subcultured when cells covered the bottom.

\section{Preparation of Dendritoma}

Dendritic cells cultured for 7 days were stained with CD86-FITC labeled and then mixed uniformly in a $2: 1$ ratio with glioma cells prestained with $\mathrm{PKH} 26$ in a centrifuge tube. Cell mix was centrifuged and the supernatant discarded. $1 \mathrm{ml}$ of $50 \%$ PEG solution was slowly added in following with one-minute incubation. The suspension mixture was then centrifuged and the supernatant discarded to terminate the fusion. Cells were resuspended in a complete culture medium, cultured for $36 \mathrm{hrs}$, and the final fused cells were collected using flow cytometry.

\section{Preparation of specific cytotoxic T lymphocytes}

$\mathrm{T}$ lymphocyte cells were collected and eluted using the nylon wool column from the previously collected non-adherent lymphocytes. Glioma tumor cells were co-cultured in the presence of DC in a ratio of $3: 1$ for 36 hours to prepare DC. The DC were then mixed with lymphocytes in a ratio of $1: 10$ for 3 days. Finally, the glioma-specific cytotoxic $\mathrm{T}$ lymphocytes (CTLs) were generated by co-culture in the presence of the fused cells in a ratio of $1: 10$ within 3 days. After generation, these CTLs were harvested as non-adherent cells, washed and added to the plates for cytotoxic assay.

\section{Cytotoxicity of specific cytotoxic $T$ lymphocytes against glioma cells}

Glioma cell suspensions were designated as three groups including (1) the CTL effective cell group acti- 
vated by fused cells; (2) the second effective cell group stimulated by co-cultured glioma cell and DC cells; and (3) only lymphocytes as a control group not stimulated by the DC. Cytotoxic T lymphocytes were plated out on a 96well culture plate and the ratios of CTLs : glioma cells were adjusted to $10: 1,20: 1,40: 1$ and $80: 1$ with three replicates, respectively. Two negative control groups were set up to include only target glioma cells without effector cells, and only effector cells. The optical densities were measured using OD after 3 days in culture via MTT assay. Data were expressed as the mean of three ODs. The effector cell killing rate (\%) was calculated using the following formula [OD1-(OD3-OD2)] / OD1 × 100, where OD1: optical density of the target cells; OD2: optical density of effective cells; OD3: optical density of target cells after effective cell reaction.

\section{Statistical analysis}

Values were shown as mean \pm SD. SPSS statistical software was used for statistical analysis.

\section{Results}

\section{Detection of dendritic cells morphology and phenotype}

Peripheral blood mononucleated cell (PBMC) collected from peripheral blood were cultured for 2 hours to obtain adherent cells (Fig. 1A). To the adherent cell cultures, cytokines were then added and the cells and cultures resumed for an additional 24 hours. Following the culture period, cell aggregation was observed as colonies transformed to present a larger phenotype, forming clusters that were suspended following 6 days in culture. At that time, the colony cell edge presented a ruffled appearance, a hallmark of DC morphology (Fig. 1B). After adding TNF- $\alpha$, cells appeared irregular upon microscopic evaluation, changing from an aggregated to a disperse state. Finally, the typical DC was observed as presenting obvious bulging surface expansion (Fig. 1C).

Upon flow cytometric evaluation, mononuclear cells were devoid of both CD86 and HLA-DR surface expression (Fig. 1D). Following culture in the presence of GMCSF and IL-4, a low expression of CD86 and HLA-DR was observed (Fig. 1E), which was consistent with the immature DC phenotype. Conversely, a higher expression of CD86 and HLA-DR was observed after addition of TNF- $\alpha$ (Fig. 1F), indicative of DC maturation and activation.

\section{Morphology of primary cultured glioma cells}

Under microscopic examination, glioma cells initially presented as round suspensions, dispersed in culture medium. After 4 hours, cells became oval and attached to the wall of the culture dish. Following 48 hours of culture, cell numbers were reduced with lower density, however cell proliferation was vigorous. Tumor cell shape and size varied, appearing as triangular, polygonal and irregular with the smaller cellular bulges. After a 96-hour incubation, cell density significantly increased, and morphology was again varied and consistent with glioma pathology; this consistent cellular morphology continued for 10 days of incubation (Fig. 2A).
A

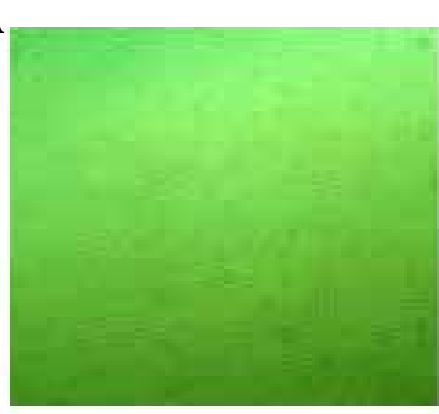

D

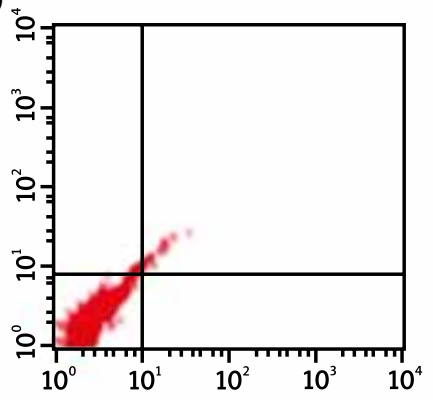

B

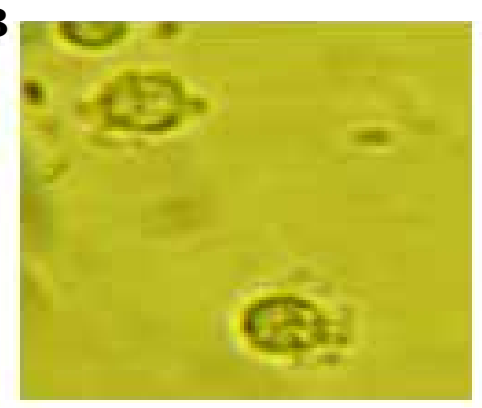

$\mathbf{E}$

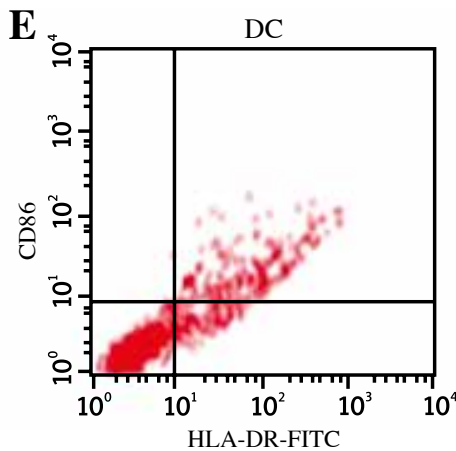

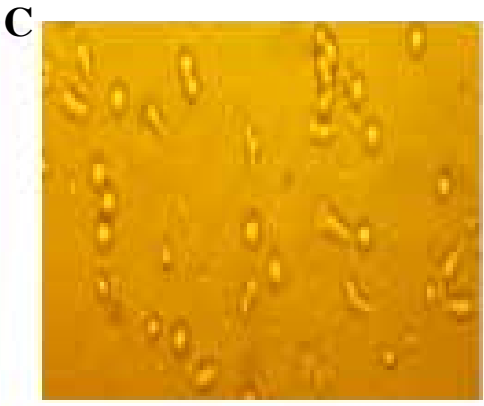

$\mathbf{F}$

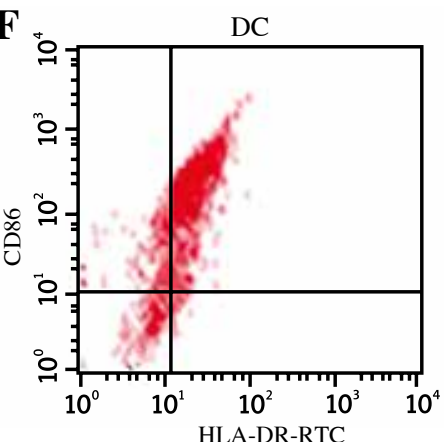

Fig. 1. 

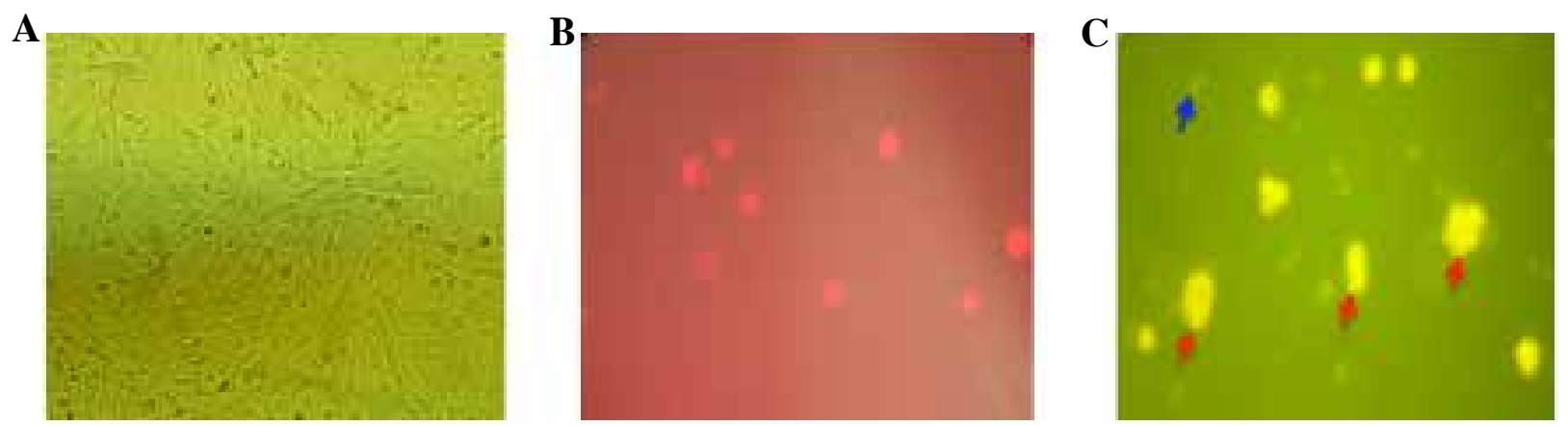

Fig. 2. A) 10-day primary cultured glioma cells; B) Glioma cell staining (PKH26 stained red); C) Dendritic cells and glioma cell fusion (DC: 200× FITC labeled CD86 dyed green (blue arrow); fused cells, yellow (200×) (red arrows)

\section{Stained glioma cells and fused cells under fluorescence microscopy}

Under bright-field, DC pre-stained with FITC-labeled CD86 were observed as green cells; similarly-stained glioma cells were observed as yellow in appearance under the same conditions. All cells appeared red at $570 \mathrm{~nm}$ (Fig. 2B). After the 24-hour DC-glioma fusion reaction, cell membranes appeared to be in contact as observed under an inverted contrast microscope. Appearance results suggest that fusions may include multiple DC fusions to multiple glioma; a single DC fused with a multiple glioma cells; or multiple glioma cells fused with multiple glioma cells in a cluster. After a 36-hour fusion reaction, DC was green and fusion cells were yellow as observed under fluorescence microscopy (Fig. 2C).

\section{Cytotoxicity test}

The killing effect of CTLs activated by fused cells was significantly higher than that of the CTL group cocultured in the presence of unsensitized lymphocytes $(p<0.01)$. The killing activity efficiency ratio was increased significantly in both fused cells and co-culture CTL groups (Table 1).

\section{Discussion}

Glioma cells naturally present with low immunogenicity potential. Studies have found that various T cells have the capacity to infiltrate inside glioma [11], and lymphocytes around glioma often serve as an indicator for a better prognosis [12]. Dendritic cells are currently known to be the most powerful of the antigen-presenting cells, with only one having the potential to initially activate $\mathrm{T}$ lymphocytes [2]. Dendritic cells also connect innate immunity and adaptive immunity as the central hub of the entire immune system. Because DC activation and function directly affect the type of immune response, DC functionality warrants more investigation to define therapeutic potential [13]. In the therapy of glioma, research has shown that DCs induce tumor-specific cytotoxic T cells. Furthermore, DC vaccines have been used in patients presenting with small lesions as part of the therapeutic regimen following surgery, radiotherapy, or chemotherapy due to their well-tolerated and induced immune response [14].

Dendritic cells are widely distributed in very low numbers in various tissues. It is therefore critical to establish a mature DC culture technique to obtain sufficient numbers of DCs for functional studies [15]. The isolation and culture of DCs in vitro requires costly, novel techniques under defined conditions [16]. Dendritic cells can be derived from cord blood, bone marrow and peripheral blood. Obtaining DCs from human bone marrow is more difficult and complex. The efficiency of DC amplification from cord blood has been shown to be significantly higher than that from peripheral blood. Dendritic cells cultured

Table 1. Cytotoxicity test of fusion cells

\begin{tabular}{|c|c|c|c|c|}
\hline \multirow[t]{2}{*}{ Group } & \multicolumn{4}{|c|}{ Ratios of CTLs to glioma cell } \\
\hline & $10: 1$ & $20: 1$ & $40: 1$ & $80: 1$ \\
\hline fusion cell & $36.15 \pm 2.17^{*, \#, \mathrm{a}}$ & $53.78 \pm 1.46^{*, \#, \mathrm{~b}}$ & $66.21 \pm 2.27^{*, \#, c}$ & $82.78 \pm 2.03^{*, \#, \mathrm{~d}}$ \\
\hline co-culture & $29.10 \pm 1.87^{*, a}$ & $37.6 \pm 1.54^{*}, \mathrm{~b}$ & $49.12 \pm 1.43^{*, c}$ & $57.49 \pm 1.62^{*, \mathrm{~d}}$ \\
\hline lymphocyte & $12.44 \pm 1.20$ & $13.70 \pm 1.66$ & $14.94 \pm 1.71$ & $16.27 \pm 1.68$ \\
\hline$p$ & 0.0001 & 0.0001 & 0.0001 & 0.0001 \\
\hline
\end{tabular}


from peripheral blood offer some advantages: 1) easily obtainable sample; 2) wide donor pool availability; 3) ease of controlling the maturation stage. Therefore, peripheral blood offers an ideally-suited method for obtaining DCs. Consequently, in the current study, selected peripheral blood was a source of DCs. Density gradient centrifugation was used in the isolation of PBMC. Red blood cells and granulocytes were isolated in lower fractions due to the greater gravities than that of lymphocytes; mononuclear cells were left in the interface of the separation medium. This method was suitable for the isolation from a small amount of blood. Higher volumes of the sample required division into several tubes; however, such repeated operation may present an increased chance of contamination.

The source, phenotype and function of DCs are both diverse and heterogeneous. Dendritic cells not only induce the activation of T cells, but also play an important role in inducing central and peripheral tolerance [17]. The immunomodulatory effects of DCs are closely related to the maturation conditions [18]. While mature dendritic cells can activate $\mathrm{T}$ lymphocytes to induce an immune response, the strong antigen processing nature of immature DCs can suppress T lymphocyte activation, resulting in inefficient $\mathrm{T}$ lymphocytes priming [19]. In vitro studies have further shown that immature DCs can disable the induction of antigen-specific $\mathrm{T}$ cells [20]. In relation, studies have correspondingly showed that the increased degree of DC maturation may be linked to the induction of efficient anti-virus-specific cytotoxic T cells [21]. To this end, studies have offered that multiple cytokines administered in a twostep combination method induce DCs harvested from peripheral blood; the initial addition of GM-CSF and IL-4 act on mononuclear cells to promote immature DC differentiation while the subsequent addition of TNF- $\alpha$ stimulate maturation of DCs.

In this study, mononuclear cells were obtained from anti-coagulated peripheral blood of glioma patients by applying lymphocyte separation medium. Mononuclear cells were induced to grow and differentiate toward DCs in culture through the addition of GM-CSF and IL-4, which inhibited the production of neutrophils and macrophages. Tumor necrosis factor $\alpha$ was then added to promote the maturation of DCs so that DCs induce a strong capacity to stimulate T cells. A typical visual DC morphology was recorded as observed using microscopy evaluation presenting characteristics of a ruffled-like bulge of the cell surface that gradually increased with cell differentiation and maturation. Flow cytometric analysis further characterized the DC maturation based upon a high expression of HLA-DR and CD86 phenotype. The morphology and proliferation were consistent with the variation observed in cultured DCs from previous in vitro studies, and provided volumes of qualified cells that could be used for further clinical study.
Specific T cell therapy may employ in vitro amplification and activation of tumor-specific $\mathrm{T}$ cells derived from cancer patients followed by re-infusion to produce a targeted immune response against the specific lesion. $\mathrm{T}$ cell sources may include the peripheral blood of patients, tumor-infiltrating lymphocytes, lymph nodes, and tumor tissues. In response to the tumor microenvironment, the host has a low endogenous anti-tumor response. To enhance the specific anti-tumor response, genetic engineering strategies have been employed to facilitate the expression of TCR on peripheral blood-derived T cells, and then can be used to recognize the tumor antigen, and thereby amplify the specific T cell response [22].

Preparation of antitumor vaccines by different methods may optimize and enhance the function of DCs in this regard. In the method of simulating maturation of DCs modified via the multi-form tumor antigens, the resulting fusion of glioma cells and autologous DCs is termed heterozygote Dendritoma. The fusion presents unique tumor-specific antigens and costimulatory effects with DCs. This specific cell has the capacity to efficiently present a large number of tumor cell-specific antigens via the structural components known to stimulate the immune response (e.g. MHC-I, class II molecules and costimulatory signal molecules) against specific and targeted tumor toxicity. Using Dendritoma to treat malignancy based on the current findings is therefore feasible [23]. Tumor vaccines composed of tumor-DC fusions not only express all of the antigens of tumor cells, but also serve in the capacity of antigen presentation and activation of T cell [3], thereby enhancing the immune response.

Cell fusion technology imparts an advantage for eliciting an effective immune response as all antigens of tumor cells are presented by the fusion cells. Considering that the majority of antigen types defining human tumor cells are yet to be clearly identified, fused tumor cells might serve as a simple and effective methodology for generating an effective antitumor vaccine. The best candidates of cells are autologous tumor cells; DCs can be derived from the patient. Current fusion methods employ the polyethylene glycol method (PEG) and electric fusion method, both of which can be applied to the clinical application. The current fusion method with the highest efficiency involves electric fusion, offering efficiency 1 to 2 grades higher than the chemical fusion method [24]. However, electric fusion methodology requires specialized equipment for operation, and is utilized with a higher expense. The polyethylene glycol method is an accessible, economical and practical methodology [25]. Purification of fusion cells, utilizing transgenic technology for selection criteria, may indeed present as a growing research area for development of a purified DC vaccine.

In summary, while DC-tumor cell fusions are currently analyzed in animal models and in assays of in vitro analysis, fusion cells offer a specific advantage for the presenta- 
tion of all the tumor cell antigens, and are a valuable method for tumor immune therapy. Our study has elucidated that a dendritic cell-glioma fusion vaccine possessed more effective anticancer activity by stimulating the effector CTLs. These results suggest that the preparation of a safe and effective fused vaccine is promising to the clinical therapy for glioma patient treatment.

\section{The authors declare no conflict of interest.}

\section{References}

1. Song Y, Liu Z, Zhong Y, et al. (2010): The effects of combined therapy of PI3K inhibitor and Ad-PTEN in human glioma nude mice model. Chin J Nervous Mental Dis 36: 104-107.

2. Banerjee D, Matthews P, Matayeva E, et al. (2008): Enhanced T-cell responses to glioma cells coated with the anti-EGF receptor antibody and targeted to activating FcgammaRs on human dendritic cells. Immunother 31: 113-120.

3. Fan F, Zhu Z (2011): Progress of dendritic cell in tumor immunotherapy. Medical Recapitulate 17: 542-544.

4. Nakahara N, Pollack IF, Storkus WJ, et al. (2003): Effective induction of antiglioma cytotoxic T cells by coadministration of interferon-beta gene vector and dendritic cells. Cancer Gene Ther 10: 549-558.

5. Dhodapkar KM, Cirignano B, Chamian F, et al. (2004): Invariant natural killer $\mathrm{T}$ cells are preserved in patients with glioma and exhibit antitumor lytic activity following dendritic cell-mediated expansion. Int J Cancer 109: 893-899.

6. Kikuchi T, Akasaki Y, Abe T, Ohno T (2002): Intratumoral injection of dendritic and irradiated glioma cells induces anti-tumor effects in a mouse brain tumor model. Cancer Immunol Immunother 51: 424-430.

7. Okada H, Kohanbash G, Zhu X, et al. (2009): Immunotherapeutic approaches for glioma. Crit Rev Immunol 29: 1-42.

8. Soling A, Rainov NG (2001): Dendritic cell therapy of primary brain tumors. Mol Med 7: 659-667.

9. Sloan AE, Parajuli P (2003): Human autologous dendritic cell-glioma fusions: feasibility and capacity to stimulate $\mathrm{T}$ cells with proliferative and cytolytic activity. J Neurooncol 64: 177-183.

10. Koido S, Homma S, Okamoto M, et al. (2013): Strategies to improve the immunogenicity of anticancer vaccines based on dendritic cell/malignant cell fusions. Oncoimmunology 2: e25994.

11. Waziri A, Killory B, Ogden AT 3rd, et al. (2008): Preferential in situ CD4+CD56+ T cell activation and expansion within human glioblastoma. J Immunol 180: 7673-7680.

12. Dunn GP, Dunn IF, Curry WT (2007): Focus on TILs: prognostic significance of tumor infiltrating lymphocytes in human glioma. Cancer Immun 7: 12.

13. Zhou C, Su Z, Zhu J, et al. (2011): Functional study of transcription factor HIx modified dendritic cell line DC 2.4. Chin J Cell Mol Immunol 27: 274-277.

14. Yu H, Zhang J, Liu Y, et al. (2010): In vitro study of cord blood antigen-sensitized dendritic cells induce anti-tumor effects. Chin J Laboratory Diagn 14: 107-108.
15. Liu Z, Dai J, Fu Z, et al. (2011): Culture and preliminary identification of murine bone marrow dendritic cells in vitro. J Biol 28: 25-27.31.

16. Li W, Chen H (2009): Immunotherapy of glioma. Chin J Minimally Invasive Neurosurg 14: 478-480.

17. Li L, Sun S, Cao X, et al. (2011): Impact of immature dendritic cells to T cell function and IL-10 and TGF- $\beta 1$ expression. Immunol J 27: 122-125.

18. Shi Q, Zeng W, Chen M, et al. (2009): Impact of IL-10 and TNF- $\alpha$ to dendritic cells in chronic hepatitis B patients. Immunol J 25: 311-313.

19. Yang M, Yang Y, Yue B, et al. (2011): Identification and Immunological Study of ImDCs cytokine-induced. J Chinese Pract Diagn Ther 25: 466-469.

20. Xia Y, Zhang Y, Jiang S, Cheng H (2010): CD4(+) T-cell anergy induced by lin(-) CD117(c-kit)(+) stem cell-derived immature dendritic cells loaded with nuclear antigen derived from Trypanosoma equiperdum. Autoimmunity 43: 664-671.

21. Shinozaki Y, Wang S, Miyazaki Y, et al. (2009): Tumor-specific cytotoxic $\mathrm{T}$ cell generation and dendritic cell function are differentially regulated by interleukin-27 during development of anti-tumor immunity. Int J Cancer 124: 1372-1378.

22. Wen Y, Gao L, Li M (2011): Interaction of cancer with immunity and the novel strategy of cancer immunotherapy. China Cancer 20: 103-107.

23. Kjaergaard J, Wang LX, Kuriyama H, et al. (2005): Active immunotherapy for advanced intracranial murine tumors by using dendritic cell-tumor cell fusion vaccines. J Neurosurg 103: $156-164$

24. Zhu C, Zheng Q, Zhang Q (2011): Application and development of dendritic cells in cancer immunotherapy. Pract J Cancer 26: 108-110.

25. Shi D, Xiong L, Qin Z, et al. (2010): Preparation of multiple gene regulated neuroglioma vaccine. Clin Med Engineering 17: 6-9. 